\title{
Mathematical modeling of groundwater contamination with varying velocity field
}

\author{
Pintu Das, Sultana Begam, Mritunjay Kumar Singh* \\ Department of Applied Mathematics, Indian Institute of Technology (Indian School of Mines), Dhanbad-826004, Jharkhand, India. \\ * Corresponding author. E-mail: drmks29@rediffmail.com
}

\begin{abstract}
In this study, analytical models for predicting groundwater contamination in isotropic and homogeneous porous formations are derived. The impact of dispersion and diffusion coefficients is included in the solution of the advection-dispersion equation (ADE), subjected to transient (time-dependent) boundary conditions at the origin. A retardation factor and zero-order production terms are included in the ADE. Analytical solutions are obtained using the Laplace Integral Transform Technique (LITT) and the concept of linear isotherm. For illustration, analytical solutions for linearly space- and time-dependent hydrodynamic dispersion coefficients along with molecular diffusion coefficients are presented. Analytical solutions are explored for the Peclet number. Numerical solutions are obtained by explicit finite difference methods and are compared with analytical solutions. Numerical results are analysed for different types of geological porous formations i.e., aquifer and aquitard. The accuracy of results is evaluated by the root mean square error (RMSE).
\end{abstract}

Keywords: Solute; Advection; Diffusion; Dispersion; Aquifer; Finite Difference Method.

\section{INTRODUCTION}

Analytical solutions of the advection-dispersion solute transport equation remain useful for a large number of applications in water science and engineering, hydrological science and engineering, environmental science and engineering etc. They are also useful to benchmark the numerical codes and solution and as a preliminary predictive tool in groundwater resource management. The bulk transport of solutes is dominated by advection and dispersion with which diffusion is usually lumped. Longitudinal dispersion causes contaminant plumes to spread out in the direction of flow. The spreading is due to the heterogeneity of the medium, such as a distribution of pore sizes and shapes. Dispersion which is perpendicular to the aquifer flow direction is called transverse dispersion. It is related to diffusion and plays an important role in the remediation of contaminants. It helps dilute the concentration of contaminants and mix them with reactive compounds. The combination of hydrodynamic dispersion and molecular diffusion coefficients is considered to describe the solute transport (Batu, 2006; Sharma and Reddy, 2004).

The dominant process of solute transport is advection moving aqueous chemical species along with fluid flow. Most of the solute transport modeling begins with advective transport. The advection-dispersion equation describes the spatial and temporal variation in solute concentration with specific initial and boundary conditions. The governing equation known as the constant-parameter advection-dispersion equation may be derived for the case of steady and unsteady flows. The traditional advection-dispersion equation represents a standard model to predict the solute concentration in an aquifer which is based on conservation of mass and Fick's law of diffusion (Bear, 1972; Fried and Combarnous, 1971).

Scheidegger (1957) explored the dispersion theory based on a relationship between two parameters (i.e., dispersion coefficient and seepage velocity) and proposed two possible relationships that (i) the dispersion coefficient is proportional to seepage velocity and (ii) the dispersion coefficient is proportional to the square of seepage velocity. A general theory of dispersion in porous media was explored by Scheidegger (1961). Later, the dispersion theory was generalized as dispersion coefficients proportional to the power of seepage velocity where power ranges from 1 to 2 (Freeze and Cherry, 1979). It has also been experimentally observed that the dispersion coefficient is directly proportional to the seepage velocity with a power ranging from 1 to 1.2 (Ghosh and Sharma, 2006).

Ebach and White (1958) described the time-dependent input concentration for longitudinal dispersion flow. Barry and Sposito (1989) obtained a closed form solution to solute transport in a semi-infinite domain with an arbitrarily time-dependent dispersion coefficient and arbitrary initial and boundary flux conditions by variable transformation. They also computed a numerical solution using a simple scheme and compared it with the analytical solution. The numerical scheme was unstable for large values of $\Delta t$. Ogata and Banks (1961) presented a solution to a one-dimensional advection-dispersion equation in a homogeneous medium in which the impact of diffusion was not included. Gelhar et al. (1992) proposed that the dispersivity is often found to be scale-dependent. Huang et al. (1996) employed general analytical solutions for one-dimensional solute transport in heterogeneous porous media with linearly increasing dispersivity. Aral and Liao (1996) studied the timedependent dispersion coefficient for contaminant transport. Basha and El-Habel (1993) presented analytical solutions with one-dimensional linear, asymptotic and exponential timedependent dispersivities in infinite domains. Huang et al. (2006) employed a parabolic distance-dependent dispersivity in a finite column. Guerrero and Skaggs (2010) analysed the analytical solution for the advection-dispersion equation with the distance-dependent coefficient using the generalised integral transform technique (GITT). Chen et al. (2012) presented a novel method for solving analytically multi-species advectivedispersive transport equations sequentially coupled with firstorder decay reactions. Singh et al. (2012) proposed one-dimensional analytical solution with temporally dependent dispersion in a homogeneous semi-infinite porous formation. Guerrero et al. (2013) analysed the diffusion type problems with transient boundary conditions with Duhamel theorem. You and Zhan (2013) studied the semi-analytical solution for solute transport in a finite column with linear asymptotic or exponen- 
tial distance-dependent dispersivities and time-dependent sources. Zamani and Bombardelli (2014) explored analytical solutions to the ADR (advection-dispersion-reaction) equation from which one can know the spatio-temporal changes in flow field and dispersivity. Van Genuchten et al. (2013) presented a series of one- and multi-dimensional solutions of the standard equilibrium advection-dispersion equation with and without terms accounting for zero-order production and first-order decay, which proved useful for simplified analyses of contaminant transport in surface water, and for mathematical verification of more comprehensive numerical transport models where the isotherm concept was not used.

In this study, we derived two novel analytical solutions for advection-dispersion equations with the impact of space- and time-dependent dispersion with diffusion. Both equations were solved analytically, subjected to linear adsorption coefficient in the solid and liquid phases. First, they were solved with respect to the space-dependent dispersion in longitudinal direction of flow with diffusion. The asymptotic dispersivity was considered which increased steadily with respect to space from the input source. The domain was initially not solute free i.e., the geological formation was not clean which means some initial background concentration existed. It was represented by a linear expression of the initial source with zero order production term which affected the solute concentration. One end of the domain, the time-dependent decreasing source was injected along with the uniform concentration of the initial source. The solute mass ejected from the other end of the domain so the flux was supposed to be zero. The concept of dispersion directly proportional to the seepage velocity, was employed (Freeze and Cherry, 1979). The Peclet number was used in the characteristic form with the space-dependent dispersivity which also affected the domain. A new time variable was used to define the old time in the longitudinal direction. A new transformation was used to reduce the advection term.

Second, we considered the time-dependent dispersion with diffusion. Here, we used the concept of dispersion as directly proportional to the ratio of the initial seepage velocity with the corresponding mean travel time. The dispersivity was defined for the positive time interval with respect to diffusion, i.e., the reactive solute was activated from the origin of source in the domain and travel along the longitudinal direction of flow.

The objective of this work was to derive an analytical solution with the help of Laplace Integral Transform Technique (LITT) for predicting contaminant concentration with distance and time. Numerical simulation for three different types of velocity expressions (i.e., exponentially decreasing, sinusoidally varying, algebraic sigmoid) with the corresponding mean travel time was done. The analytical solutions were analysed for the aquifer and aquitard formations. In general, the transmission capacity is low in aquitard as compared to the aquifer. So, the rate of solute concentration was high in the aquitard as compared to the aquifer. The mean travel time and Peclet number were also compared.

\section{MATHEMATICAL FORMULATION OF THE PROBLEM}

Considering a system in which non-reactive solute transport is primarily one-dimensional, i.e., solute concentrations are horizontally and vertically well-mixed so that concentrations vary only in the longitudinal or downstream direction. The effects of dispersion are constant or the time-dependent with respect to distance; and the solutes are conservative in the unsteady field. Given these assumptions, the conservation of mass yields the constant parameter advection-dispersion equation (ADE) as

$$
\frac{\partial c}{\partial t}+\frac{1-n}{n} \frac{\partial F}{\partial t}=\frac{\partial}{\partial x}\left(D \frac{\partial c}{\partial x}-u c\right)-\mu c
$$

where $c\left[\mathrm{ML}^{-3}\right]$ is the volume averaged dispersing solute concentration in the liquid phase, $F\left[\mathrm{ML}^{-3}\right]$ is the volume averaged dispersing solute concentration in the solid phase, $\mathrm{D}\left[\mathrm{L}^{2} \mathrm{~T}^{-1}\right]$ is the longitudinal dispersion coefficient (i.e. representing longitudinal dispersion), $u\left[\mathrm{LT}^{-1}\right]$ is the unsteady uniform downward pore seepage velocity, $x[\mathrm{~L}]$ is the length in longitudinal direction of flow , $t[\mathrm{~T}]$ is time, $\mu\left[\mathrm{T}^{-1}\right]$ is the first order decay rate coefficient for solute production in the liquid phase, and $n$ is the porosity of the different geological formations.

A linear adsorption coefficient in terms of the solid and liquid phases is considered as

$$
F=K_{d} c
$$

where $K_{d}$ is the distribution coefficient.

Using equation (2) in equation (1), we have

$$
R \frac{\partial c}{\partial t}=\frac{\partial}{\partial x}\left(D \frac{\partial c}{\partial x}-u c\right)-\mu c
$$

where $R=1+\frac{1-n}{n} K_{d}$

is the retardation factor.

The retardation commonly describes a reversible sorption process, including adsorption and ion exchange. Sorption is experimentally defined by the measured quantity of a solute that can be sorbed by a particular sediment, soil or rock.

Initially the aquifer is assumed to be contaminated (i.e., some initial background concentration exists in the aquifer) and it is represented by a linear combination of some kind of initial concentration and the zero order production term with seepage velocity. The mathematical expression for the assumption can be written as

$c(x, 0)=c_{i}+\frac{\gamma x}{u}, x>0, t=0$

where $c_{i}\left[\mathrm{ML}^{-3}\right]$ is the initial background concentration, and $\gamma\left[\mathrm{ML}^{-3} \mathrm{~T}^{-1}\right]$ is the zero order production rate coefficient for solute production in the liquid phase.

Solute transport is also affected by the boundary condition chosen as input time-dependent source concentration with initial background concentration at the origin i.e., at $x=0$. To solve ADE given in equation (1), an inlet boundary condition is prescribed as

$c(0, t)=c_{i}+c_{0} \exp (-\lambda t) \quad t>0, x=0$

where $\lambda\left[\mathrm{T}^{-1}\right]$ is the decay rate constant.

At the other end of the aquifer, solute transport may not be affected and therefore, an outlet boundary condition is prescribed as a no flux boundary condition, i.e.

$$
\frac{\partial c}{\partial x}=0, x \rightarrow \infty
$$




\section{Analytical solution for space-dependent dispersion with diffusion}

Let $\quad u=u_{0} f(m t), \quad D=D_{0} f(m t), \mu=\mu_{0} f(m t) \quad$ and $\gamma=\gamma_{0} f(m t)$ where $f(m t)$ is non-dimensional, and $m\left[\mathrm{~T}^{-1}\right]$ is the flow resistance coefficient. Here $D_{0}\left[\mathrm{~L}^{2} \mathrm{~T}^{-1}\right]$ is the initial longitudinal dispersion coefficient, $u_{0}\left[\mathrm{LT}^{-1}\right]$ is the initial seepage velocity, $\mu_{0}\left[\mathrm{~T}^{-1}\right]$ is the initial first order decay term, and $\gamma_{0}\left[\mathrm{ML}^{-3} \mathrm{~T}^{-1}\right]$ is the initial zero-order production term.

Equation (3) can be written as

$\frac{R}{f(m t)} \frac{\partial c}{\partial t}=\frac{\partial}{\partial x}\left(D_{0} \frac{\partial c}{\partial x}-u_{0} c\right)-\mu_{0} c$

Further we introduce a new time variable (Crank, 1975)

$T^{*}=\int_{0}^{t} f(m t) d t$

Using equation (9) in equation (8) we obtain

$R \frac{\partial c}{\partial T^{*}}=\frac{\partial}{\partial x}\left(D_{0} \frac{\partial c}{\partial x}-u_{0} c\right)-\mu_{0} c$

The dispersion coefficient is expressed as a combination of hydrodynamic dispersion and diffusion as

$D_{0}=q x u_{0}+D^{*}$

where $q$ is the non-dimensional constant parameter growing rate of dispersivity w. r. t. distance, and $D^{*}\left[\mathrm{~L}^{2} \mathrm{~T}^{-1}\right]$ is the molecular diffusion coefficient. In most of the previous works, the impact of diffusion is not considered because it does not vary significantly for different soil and contaminant combinations and ranges from $1 \times 10^{-9}$ to $2 \times 10^{-9} \mathrm{~m}^{2} / \mathrm{sec}$ (Mitchell, 1976). Later, it was found that even a small value for the diffusion coefficient may also have significant impact on solute transport in porous media, especially for fine-grained geologic materials (Gillham and Cherry, 1982).
Now applying equation (11) in equation (10) we have

$R \frac{\partial c}{\partial T^{*}}=\left(q x u_{0}+D^{*}\right) \frac{\partial^{2} c}{\partial x^{2}}-u_{0}(1-q) \frac{\partial c}{\partial x}-\mu_{0} c$

Applying the following non-dimensional parameters

$$
C=\frac{c}{c_{0}}, X=\frac{x u_{0}}{D_{0}}, T=\frac{T^{*} u_{0}^{2}}{D_{0}}, \mu_{1}=\frac{\mu_{0} D_{0}}{u_{0}^{2}}, \gamma_{1}=\frac{\gamma_{0} D_{0}}{c_{0} u_{0}^{2}}
$$

in equation (12), one can get as

$$
\begin{aligned}
& R \frac{\partial C}{\partial T}=D_{1} \frac{\partial^{2} C}{\partial X^{2}}-A_{1} \frac{\partial C}{\partial X}-\mu_{1} C \\
& D_{1}=\frac{\alpha_{0} u_{0}}{D_{0}}+\frac{D^{*}}{D_{0}}, A_{1}=1-\frac{1}{P e}, \text { and } P e=\frac{x}{\alpha_{0}}, \alpha_{0}=q x
\end{aligned}
$$

where $\alpha_{0}$ is asymptotic dispersivity.

Now the initial and boundary conditions are expressed in terms of non-dimensional parameters as

$$
\begin{aligned}
& C(X, 0)=\frac{c_{i}}{c_{0}}+\gamma_{1} X, \quad X>0, T=0 \\
& C(0, T)=\frac{c_{i}}{c_{0}}+1-\lambda^{*} T, \quad X=0, T>0, \text { where } \lambda^{*}=\frac{\lambda D_{0}}{u_{0}{ }^{2}} \\
& \frac{\partial C}{\partial X}=0, \quad X \rightarrow \infty, T \geq 0
\end{aligned}
$$

We used the transformation

$$
C(X, T)=K(X, T) \exp \left[\frac{A_{1}}{2 D_{1}}-\frac{1}{R}\left(\frac{A_{1}^{2}}{4 D_{1}}+\mu_{1}\right) T\right]
$$

to eliminate the convective terms form of equation (14) where $K(X, T)$ is another dependent variable.

Using equation (19) for reducing the advective term from equation (14) and analytical solution obtained subject to initial and boundary conditions given in equations (16) - (18) by applying the Laplace transform technique, the solution can be written as

$$
\left.C(X, T)=\left[\begin{array}{l}
\left(\frac{c_{i}}{c_{0}}+1\right) F(X, T)-\lambda^{*} G(X, T)-\frac{c_{i}}{c_{0}} H(X, T) \\
+\frac{\gamma_{1} A_{1}}{R} I(X, T)+\left(\frac{c_{i}}{c_{0}}+\gamma_{1} X-\frac{\gamma_{1} A_{1}}{R} T\right) e^{-\left(\frac{A_{1}}{2 D_{1}} X-\frac{A_{1}^{2}}{4 D_{1} R} T\right)}
\end{array}\right] \exp \left[\frac{A_{1}}{2 D_{1}}-\frac{1}{R}\left(\frac{A_{1}^{2}}{4 D_{1}}+\mu_{1}\right)\right] T\right]
$$

where $F(X, T)=\frac{1}{2}\left[\exp \left(\beta T-\sqrt{\frac{\beta R}{D_{1}}} X\right) \operatorname{erfc}\left(\frac{1}{2} \sqrt{\frac{R}{D_{1} T}} X-\sqrt{\beta T}\right)+\exp \left(\beta T+\sqrt{\frac{\beta R}{D_{1}}} X\right) \operatorname{erfc}\left(\frac{1}{2} \sqrt{\frac{R}{D_{1} T}} X+\sqrt{\beta T}\right)\right]$

$$
\begin{aligned}
G(X, T) & =\frac{1}{4 \sqrt{\beta}}\left[2 \sqrt{\beta} T-\sqrt{\frac{R}{D_{1}}} X\right] \exp \left(\beta T-\sqrt{\frac{\beta R}{D_{1}}} X\right) \operatorname{erfc}\left(\frac{1}{2} \sqrt{\frac{R}{D_{1} T}} X-\sqrt{\beta T}\right) \\
& +\frac{1}{4 \sqrt{\beta}}\left[2 \sqrt{\beta} T+\sqrt{\frac{R}{D_{1}}} X\right] \exp \left(\beta T+\sqrt{\frac{\beta R}{D_{1}}} X\right) \operatorname{erfc}\left(\frac{1}{2} \sqrt{\frac{R}{D_{1} T}} X+\sqrt{\beta T}\right)
\end{aligned}
$$




$$
\begin{aligned}
H(X, T) & =\frac{1}{2}\left[\exp \left(\frac{A_{1}^{2}}{4 D_{1} R} T-\frac{A_{1}}{2 D_{1}} X\right) \operatorname{erfc}\left(\frac{1}{2} \sqrt{\frac{R}{D_{1} T}} X-\frac{A_{1}}{2} \sqrt{\frac{T}{D_{1} R}}\right)+\exp \left(\frac{A_{1}^{2}}{4 D_{1} R} T+\frac{A_{1}}{2 D_{1}} X\right) \operatorname{erfc}\left(\frac{1}{2} \sqrt{\frac{R}{D_{1} T}} X+\frac{A_{1}}{2} \sqrt{\frac{T}{D_{1} R}}\right)\right] \\
I(X, T) & =\frac{\sqrt{D_{1}}}{2 A_{1}}\left(\frac{A_{1} T}{\sqrt{D_{1} R}}-\sqrt{\frac{R}{D_{1}}} X\right) \exp \left(\frac{A_{1}^{2}}{4 D_{1} R} T-\frac{A_{1}}{2 D_{1}} X\right) \operatorname{erfc}\left(\frac{1}{2} \sqrt{\frac{R}{D_{1} T}} X-\frac{A_{1}}{2} \sqrt{\frac{T}{D_{1} R}}\right) \\
+ & \frac{\sqrt{D_{1}}}{2 A_{1}}\left(\frac{A_{1} T}{\sqrt{D_{1} R}}+\sqrt{\frac{R}{D_{1}}} X\right) \exp \left(\frac{A_{1}^{2}}{4 D_{1} R} T+\frac{A_{1}}{2 D_{1}} X\right) \operatorname{erfc}\left(\frac{1}{2} \sqrt{\frac{R}{D_{1} T}} X+\frac{A_{1}}{2} \sqrt{\frac{T}{D_{1} R}}\right)
\end{aligned}
$$

and $\beta=\frac{1}{R}\left(\frac{A_{1}^{2}}{4 D_{1}}+\mu_{1}\right)$

\section{Analytical solution for time-dependent dispersion with diffusion}

In this case, we use the concept of dispersion which is directly proportional to the initial seepage velocity and inversely proportional to the mean travel time of the solute transported through the aquifer. Let $u=u_{0} \frac{f(m t)}{K_{1}}, \quad D=D_{0} \frac{f(m t)}{K_{1}}$, $\mu=\frac{\mu_{0}^{*} f(m t)}{K_{1}}$ and $\gamma=\frac{\gamma_{0}^{*} f(m t)}{K_{1}}$ where $K_{1}$ is the mean travel time $\mu_{0}^{*}\left[\mathrm{~T}^{-1}\right]$ is the initial first order decay rate coefficient for solute production in the liquid phase, and $\gamma_{0}^{*}\left[\mathrm{ML}^{-3} \mathrm{~T}^{-1}\right]$ is the initial zero-order production term.

Now equation (3) can be written as

$R \frac{K_{1}}{f(m t)} \frac{\partial c}{\partial t}=\frac{\partial}{\partial x}\left(D_{0} \frac{\partial c}{\partial x}-u_{0} c\right)-\mu_{0}^{*} c$

Further, using the new time-dependent variable

$\tau^{*}=\int_{o}^{t} \frac{f(m t)}{K_{1}} d t$

in the above equation (22), we get as follows:

$R \frac{\partial c}{\partial \tau^{*}}=\frac{\partial}{\partial x}\left(D_{0} \frac{\partial c}{\partial x}-u_{0} c\right)-\mu_{0}^{*} c$

where $D_{0}$ is the dispersion coefficients defined in terms of the linear time-dependent expression

$$
D_{0}=Q \frac{f(m t)}{K_{1}}+D^{*}
$$

where $Q$ is the maximum dispersivity, $K_{1}$ is the mean travel time, $D^{*}$ is the molecular diffusion coefficients, and $f(m t)$ is the generalised case of the time-dependent function which describes the diffusivity in the whole domain.

Since the mean travel distance for the dispersivity is directly proportional to the mean travel time in the longitudinal flow with a constant velocity field, the two formulations for the diffusivity are considered in the equilibrium and nonequilibrium forms.
The value of maximum dispersivity $Q$ for the timedependent diffusion in the semi-infinite domain is considered as follows:

$$
Q=\left\{\begin{array}{cc}
\frac{K_{1}}{f(m t)} ; & \forall t>0 \\
0 ; & \forall t<0
\end{array}\right\}
$$

Using equation (25) in the equation (24), we have

$$
R \frac{\partial c}{\partial \tau^{*}}=\left[Q \frac{f(m t)}{K_{1}}+D^{*}\right] \frac{\partial^{2} c}{\partial x^{2}}-u_{0} \frac{\partial c}{\partial x}-\mu_{0}^{*} c
$$

For the positive value of time the equation (27) reduces to

$$
\begin{aligned}
& R \frac{\partial c}{\partial \tau^{*}}=\left[1+D^{*}\right] \frac{\partial^{2} c}{\partial x^{2}}-u_{0} \frac{\partial c}{\partial x}-\mu_{0}^{*} c \\
& \text { i.e., } R \frac{\partial c}{\partial \tau^{*}}=D^{*} \frac{\partial^{2} c}{\partial x^{2}}-u_{0} \frac{\partial c}{\partial x}-\mu_{0}^{*} c
\end{aligned}
$$

Again using the non-dimensional variable we have

$$
C=\frac{c}{c_{0}}, X=\frac{x u_{0}}{D_{0}}, \tau=\frac{\tau^{*} u_{0}^{2}}{D_{0}}, \mu_{2}=\frac{\mu_{0}^{*} D_{0}}{u_{0}^{2}}, \gamma_{2}=\frac{\gamma_{0}^{*} D_{0}}{c_{0} u_{0}^{2}}
$$

Equation (28) reduces to

$R \frac{\partial C}{\partial \tau}=D_{11} \frac{\partial^{2} C}{\partial X^{2}}-\frac{\partial C}{\partial X}-\mu_{2} C$, where $D_{11}=\frac{D_{1}^{*}}{D_{0}}$

The corresponding initial and boundary conditions in terms of the non-dimensional variable, can be written as

$C(X, 0)=\frac{c_{i}}{c_{0}}+\gamma_{2} X, X>0, \tau=0$

$C(0, \tau)=\frac{c_{i}}{c_{0}}+1-\lambda^{*} \tau, X=0, \tau>0$, where $\lambda^{*}=\frac{\lambda D_{0}}{u_{0}^{2}}$

$\frac{\partial C}{\partial X}=0, \quad X \rightarrow \infty, \tau \geq 0$

Further using the following transformation

$$
C(X, \tau)=K(X, \tau) \exp \left[\frac{1}{2 D_{11}} X-\frac{1}{R}\left(\frac{1}{4 D_{11}}+\mu_{2}\right) \tau\right]
$$

from the above equations (30) - (33) and by applying the same procedure as discussed earlier, we get the solution as 


$$
\begin{aligned}
& C(X, \tau)=\left[\begin{array}{l}
\left(\frac{c_{i}}{c_{0}}+1\right) F_{1}(X, \tau)-\lambda^{*} G_{1}(X, \tau)-\frac{c_{i}}{c_{0}} H_{1}(X, \tau) \\
+\frac{\gamma_{2}}{R} I_{1}(X, \tau)+\left(\frac{c_{i}}{c_{0}}+\gamma_{2} X-\frac{\gamma_{2}}{R} T\right) e^{-\left(\frac{1}{2 D_{11}} X-\frac{1}{4 D_{11} R} \tau\right)}
\end{array}\right] \exp \left[\frac{1}{2 D_{11}} X-\frac{1}{R}\left(\frac{1}{4 D_{11}}+\mu_{2}\right) \tau\right] \\
& \text { where } F_{1}(X, \tau)=\frac{1}{2}\left[\exp \left(\xi \tau-\sqrt{\frac{\xi R}{D_{11}}} X\right) \operatorname{erfc}\left(\frac{1}{2} \sqrt{\frac{R}{D_{11} \tau}} X-\sqrt{\xi \tau}\right)+\exp \left(\xi \tau+\sqrt{\frac{\xi R}{D_{11}}} X\right) \operatorname{erfc}\left(\frac{1}{2} \sqrt{\frac{R}{D_{11} \tau}} X+\sqrt{\xi \tau}\right)\right] \\
& G_{1}(X, \tau)=\frac{1}{4 \sqrt{\xi}}\left[2 \sqrt{\xi} \tau-\sqrt{\frac{R}{D_{11}}} X\right] \exp \left(\xi \tau-\sqrt{\frac{\xi R}{D_{11}}} X\right) \operatorname{erfc}\left(\frac{1}{2} \sqrt{\frac{R}{D_{11} \tau}} X-\sqrt{\xi \tau}\right) \\
& +\frac{1}{4 \sqrt{\xi}}\left[2 \sqrt{\xi} \tau+\sqrt{\frac{R}{D_{11}}} X\right] \exp \left(\xi \tau+\sqrt{\frac{\xi R}{D_{11}}} X\right) \operatorname{erfc}\left(\frac{1}{2} \sqrt{\frac{R}{D_{11} \tau}} X+\sqrt{\xi \tau}\right) \\
& H_{1}(X, \tau)=\frac{1}{2}\left[\begin{array}{l}
\exp \left(\frac{1}{4 D_{11} R} \tau-\frac{1}{2 D_{11}} X\right) \operatorname{erfc}\left(\frac{1}{2} \sqrt{\frac{R}{D_{11} \tau}} X-\frac{1}{2} \sqrt{\frac{\tau}{D_{11} R}}\right) \\
+\exp \left(\frac{1}{4 D_{11} R} \tau+\frac{1}{2 D_{11}} X\right) \operatorname{erfc}\left(\frac{1}{2} \sqrt{\frac{R}{D_{11} \tau}} X+\frac{1}{2} \sqrt{\frac{\tau}{D_{11} R}}\right)
\end{array}\right] \\
& I_{1}(X, \tau)=\frac{\sqrt{D_{11} R}}{2}\left(\frac{\tau}{\sqrt{D_{11} R}}-\sqrt{\frac{R}{D_{11}}} X\right) \exp \left(\frac{1}{4 D_{11} R} \tau-\frac{1}{2 D_{11}} X\right) \operatorname{erfc}\left(\frac{1}{2} \sqrt{\frac{R}{D_{11} \tau}} X-\frac{1}{2} \sqrt{\frac{\tau}{D_{11} R}}\right) \\
& +\frac{\sqrt{D_{11} R}}{2}\left(\frac{\tau}{\sqrt{D_{11} R}}+\sqrt{\frac{R}{D_{11}}} X\right) \exp \left(\frac{1}{4 D_{11} R} \tau+\frac{1}{2 D_{11}} X\right) \operatorname{erfc}\left(\frac{1}{2} \sqrt{\frac{R}{D_{11} \tau}} X+\frac{1}{2} \sqrt{\frac{\tau}{D_{11} R}}\right)
\end{aligned}
$$

and $\xi=\frac{1}{R}\left(\frac{1}{4 D_{11}}+\mu_{2}\right)$

\section{NUMERICAL SOLUTION}

Numerical dispersion was first quantified by Lantz (1971). The second-order error was investigated through the truncated Taylor series approximation by using the explicit finite difference method to solve one-dimensional advection dispersion equation (Chaudhari, 1971). Numerical studies explored the effect of numerical dispersion (De Smedt and Wierenga, 1977; Dudley et al., 1991; van Genuchten and Gray, 1978). The root mean square was used to calculate the average error at each nodal point of the grid (Roberts and Selim, 1984). Moldrup et al. (1994) presented an explicit finite-difference model based on an unsteady one-dimensional solute transport equation. In general, the solution of the transport equation can be obtained by numerical integration. The objective of this study is to validate the analytical solution with a numerical solution for solute transport in a homogeneous porous medium with space- and time-dependent dispersion and diffusion.

\section{Numerical solution for space-dependent dispersion and diffusion}

In order to solve the advective-dispersive equation by an explicit finite difference method, the semi-infinite medium is changed into a finite medium by using the following transformation:

$$
X^{\prime}=1-\exp (-X)
$$

Using equation (37) in equation (14) we have

$R \frac{\partial C}{\partial T}=D_{1}\left(1-X^{\prime}\right)^{2} \frac{\partial^{2} C}{\partial X^{\prime 2}}-D_{2}\left(1-X^{\prime}\right) \frac{\partial C}{\partial X^{\prime}}-\mu_{1} C$

where $D_{2}=D_{1}+A_{1}$.

Initial and boundary conditions are transformed as follows:

$$
\begin{aligned}
& C\left(X^{\prime}, 0\right)=\frac{c_{i}}{c_{0}}+\gamma_{1} \log \left(\frac{1}{1-X^{\prime}}\right) ; X^{\prime}>0, T=0 \\
& C(0, T)=\frac{c_{i}}{c_{0}}+1-\lambda^{*} T ; X^{\prime}=0, T>0 \\
& \frac{\partial C}{\partial X^{\prime}}=0 ; X^{\prime}=1, T>0
\end{aligned}
$$

The one-dimensional solute transport equation represents a partial differential equation of parabolic type in which, finite difference technique is commonly used to obtain numerical solution. An explicit finite difference technique is commonly used, even though it requires extended computing time because of its restrictive stability criteria. The finite difference technique is derived by using Taylor's expansion (Carnahan et al., 1969; Mickley et al., 1957) in which partial derivatives are approximated and hence, solutions can be obtained by using the first, second or higher order accurate methods. Here, we have used the general form of an explicit finite difference approximation with forward time and central space forward difference scheme in equation (38) together with initial and boundary conditions given in equations (39), 40(a) to 40(b) which are approximated, respectively, as follows: 


$$
\begin{aligned}
C_{i, j+1}= & \left(1-\frac{\mu_{1}}{R} \Delta T\right) C_{i, j}+\frac{D_{1}}{R}\left(1-X_{i}^{\prime}\right)^{2}\left(C_{i+1, j}-2 C_{i, j}+C_{i-1, j}\right) \frac{\Delta T}{\Delta X^{\prime 2}} \\
& -\frac{D_{2}}{R}\left(1-X_{i}^{\prime}\right)\left(C_{i+1, j}-C_{i-1, j}\right) \frac{\Delta T}{2 \Delta X^{\prime}} \\
C_{i, 0}= & \frac{c_{i}}{c_{0}}+\gamma_{1} \log \left(\frac{1}{1-X_{i}^{\prime}}\right) ; \quad i>0 \\
C_{0, j}= & \frac{c_{i}}{c_{0}}+1-\lambda^{*} T_{j} ; \quad j>0 \\
C_{M, j}= & C_{M-1, j}
\end{aligned}
$$

where $i$ is the longitudinal space subscript, $j$ gives different time levels for which the solutions are approximated, $\Delta X^{\prime}$ is the increment in the longitudinal direction, and $\Delta T$ is the time increment in equation (41).

The space domain $X^{\prime}$ and time domain $T$ are discretized by a rectangular grid of point $\left(X_{i}^{\prime}, T_{j}\right)$ with mesh-size $\Delta X^{\prime}$ and $\Delta T$, respectively:

$X_{i}^{\prime}=X_{i-1}^{\prime}+\Delta X^{\prime}, i=1,2, \ldots, M, X_{0}^{\prime}=0, \Delta X^{\prime}=0.01$

$T_{j}=T_{j-1}+\Delta T, \mathrm{j}=1,2, \ldots, I, T_{0}=0, \Delta T=0.0001$

The contaminant concentration at a point for space $X_{i}^{\prime}$ with $j^{\text {th }}$ subinterval of time $T$ is defined by $C_{i, j}$.

\section{Stability analysis}

A finite difference method is convergent if the discretization error approaches zero, as the mesh sizes in time and spatial domain i.e., $\Delta T$ and $\Delta X^{\prime}$, respectively, tend toward zero (Carnahan et al.,1969). Here, we used the forward difference in time for the first order derivative with respect to time which contains the first order accuracy. The stability condition of the finite difference scheme is determined using the matrix method proposed by Smith (1978) and this technique was used by Notodarmojo et al. (1991). The finite difference scheme of the governing partial differential equation can be rewritten as follows:

$$
C_{i, j+1}=(\beta+\xi) C_{i-1, j}+(\alpha-2 \beta) C_{i, j}+(\beta-\xi) C_{i+1, j}
$$

where $\alpha=1-\frac{\mu_{1}}{R} \Delta T, \beta=\frac{D_{1}}{R} \frac{\Delta T}{\Delta X^{\prime 2}}$ and $\xi=\frac{D_{2}}{R} \frac{\Delta T}{2 \Delta X^{\prime}}$

In the matrix form, it can be written as follows:

$$
[C]^{j+1}=A[C]^{j}
$$

where matrix $A$ contains all the constants.

The difference equation is stable, if the eigenvalues of $A$ must have modulus values less than or equal to unity, i.e., $|a| \leq 1$, where $a$ is the eigenvalue of the matrix $A$.

On applying the Gerschgorin circle method to determine the bounds of eigenvalues of the matrix $A$, the stability criterion for the time step is obtained as follows:

$$
\Delta T \leq \frac{1}{\left(\frac{\mu_{1}}{2 R}+\frac{2 D_{1}}{R \Delta X^{\prime 2}}\right)}
$$

\section{Numerical solution for time-dependent dispersion with diffusion}

Applying the transformation defined in equation (37), the time-dependent diffusion equation (30) expressed in finite domain is written as follows:

$$
R \frac{\partial C}{\partial \tau}=D_{11}\left(1-X^{\prime}\right)^{2} \frac{\partial^{2} C}{\partial X^{\prime 2}}-D_{12}\left(1-X^{\prime}\right) \frac{\partial C}{\partial X^{\prime}}-\mu_{2} C
$$

where $D_{12}=1+D_{11}$.

The corresponding initial and boundary conditions are written as follows:

$$
\begin{aligned}
& C\left(X^{\prime}, 0\right)=\frac{c_{i}}{c_{0}}+\gamma_{2} \log \left(\frac{1}{1-X^{\prime}}\right) ; \quad X^{\prime}>0, \tau=0 \\
& C(0, \tau)=\frac{c_{i}}{c_{0}}+1-\lambda^{*} \tau ; \quad X^{\prime}=0, \tau>0 \\
& \frac{\partial C}{\partial X^{\prime}}=0 ; X^{\prime}=1, \tau>0
\end{aligned}
$$

Similarly, we have used the general form of the explicit finite difference approximation with forward time and central space forward difference scheme applied in equation (47) together with the initial and boundary conditions given in equations (48), 49(a) to 49(b) which are approximated, respectively, as follows:

$$
\begin{aligned}
C_{i, j+1}= & \left(1-\frac{\mu_{2}}{R} \Delta \tau\right) C_{i, j} \frac{D_{11}}{R}\left(1-X_{i}^{\prime}\right)^{2}\left(C_{i+1, j}-2 C_{i, j}+C_{i-1, j}\right) \frac{\Delta \tau}{\Delta X^{\prime 2}} \\
& -\frac{D_{12}}{R}\left(1-X_{i}^{\prime}\right)\left(C_{i+1, j}-C_{i-1, j}\right) \frac{\Delta \tau}{2 \Delta X^{\prime}} \\
C_{i, 0}= & \frac{c_{i}}{c_{0}}+\gamma_{2} \log \left(\frac{1}{1-X_{i}^{\prime}}\right) \quad i>0 \\
C_{0, j}= & \frac{c_{i}}{c_{0}}+1-\lambda^{*} \tau_{j} \\
C_{M, j}= & C_{M-1, j}
\end{aligned}
$$

where superscript $j$ refers to time; subscript $i$ refers to space, $\Delta \tau$ is the time increment, and $\Delta X^{\prime}$ is the space increment in equation (50).

The space domain $X^{\prime}$ and time domain $\tau$ are discretized by a rectangular grid of points $\left(X_{i}^{\prime}, \tau_{j}\right)$ with mesh-size $\Delta X^{\prime}$ and $\Delta \tau$, respectively:

$$
\begin{aligned}
& X_{i}^{\prime}=X_{i-1}^{\prime}+\Delta X^{\prime}, i=1,2, \ldots, M, X_{0}^{\prime}=0, \Delta X^{\prime}=0.05 \\
& \tau_{j}=\tau_{j-1}+\Delta \tau, \mathrm{j}=1,2, \ldots, I, \tau_{0}=0, \Delta \tau=0.0001
\end{aligned}
$$

The contaminant concentration at a point for the space $X_{i}^{\prime}$ with $j^{\text {th }}$ subinterval of time $\tau$ denoted by $C_{i, j}$.

\section{Stability analysis}

In a similar approach used earlier, the finite difference scheme of the governing partial differential equation (50) can be rewritten as follows: 
$C_{i, j+1}=\left(\beta_{1}+\xi_{1}\right) C_{i-1, j}+\left(\alpha_{1}-2 \beta_{1}\right) C_{i, j}+\left(\beta_{1}-\xi_{1}\right) C_{i+1, j}$

where $\alpha_{1}=1-\frac{\mu_{2}}{R} \Delta \tau, \beta_{1}=\frac{D_{11}}{R} \frac{\Delta \tau}{\Delta X^{\prime 2}}$ and $\xi_{1}=\frac{D_{12}}{R} \frac{\Delta \tau}{2 \Delta X^{\prime}}$.

In matrix form, it can be written as follows:

$[C]^{j+1}=B[C]^{j}$

where matrix $B$ contains all the constants.

On applying the same Gerschgorin circle method to determine the bounds of eigenvalues of matrix $B$, the stability criterion for the time step is obtained as follows:

$$
\Delta \tau \leq \frac{1}{\left(\frac{\mu_{2}}{2 R}+\frac{2 D_{11}}{R \Delta X^{\prime 2}}\right)}
$$

\section{Accuracy}

To assess the accuracy of the solution, we compare the numerical solution to the analytical one. The accuracy is the degree of similarity of concentration values of the analytical result to that of the numerical result. We found that the solutions were convergent, if the stability criterion was fulfilled. Towler and Yang (1979) adopted the criterion of comparison which was more systematic and consistent: (1) the RMSE (Root-Mean-Square Error) and (2) the absolute maximum error between the analytical solution and the numerical solution at all grid points. For testing the accuracy of the solutions obtained in the present work, we used RMSE which is one of the most commonly used measures to check the accuracy. The root mean square was used to calculate the average error at each point which is defined as follows:

$\operatorname{RMSE}=\sqrt{\frac{1}{N} \sum_{i=1}^{N}\left|\Delta C_{i}\right|^{2}}$

where $\Delta C=C_{\text {analytical }}-C_{\text {numerical }}$.

Here, the number of data denoted by $N$ and the difference between analytical and numerical values is denoted by $\Delta C$.

\section{NUMERICAL SIMULATION AND DISCUSSION}

This work considers the above derived solution of scaledependent and time-dependent dispersion with diffusion. The sensitivities to different parameters included in these solutions were explored. For the sake of simplicity, the contaminant behaviour predicted in the different geological formations considered three different types of velocity patterns: 1) exponentially decreasing form, 2) sinusoidally varying form and 3) algebraic sigmoid form. These velocity patterns usually exist in the rainy season and reaches up to a certain limit over the period of time. Mathematically, the velocity expressions and their corresponding new time variable are derived as follows:

1) Exponential decreasing: $u=u_{0} \exp \left(-K_{1} m t\right)$ and $T=\frac{u_{0}^{2}}{K_{1} m D_{0}}\left[1-e^{-K_{1} m t}\right]$

2) Sinusoidal form: $u=u_{0}\left[4 K_{1} \pi+m \cos \left(K_{1} m t\right)\right]$ and $T=\frac{u_{0}^{2}}{D_{0}}\left[4 K_{1} \pi t+\frac{1}{K_{1}} \sin \left(K_{1} m t\right)\right]$
3) Algebraic sigmoid: $u=u_{0}\left[\frac{m t}{\sqrt{(m t)^{2}+K_{1}^{2}}}\right]$ and $T=\frac{u_{0}^{2}}{m D_{0}}\left[\sqrt{(m t)^{2}+K_{1}^{2}}-K_{1}\right]$

where $m$ is the flow resistance coefficient and $K_{1}$ is the mean travel time corresponding to dispersion and diffusion. The first two have been used by Aral and Liao (1996) and Singh et al. (2009) without an impact of mean travel time and the last one is based on the properties of an algebraic sigmoid function which includes the error function. The sinusoidal form of the velocity pattern represents the nature of groundwater contamination in the tropical region in which the fluctuation behavior of groundwater recharge is shown. But the exponentially decreasing form of velocity shows a decreasing nature of contaminant concentration (Jain et al., 2007; Singh and Singh, 2001). The algebraic sigmoid form includes the error function. It starts to progress from a small beginning, accelerates in the rainy season, and then reaches a limit over a period of time.

For graphical representation of the analytical solution given in equation (20), the set of input values was given as $c_{i}=0.01, c_{0}=1.0, \mu_{0}=0.0001$ year $^{-1}, u_{0}=0.01 \mathrm{~km} /$ year,

$D_{0}=0.1 \mathrm{~km}^{2} /$ year, $\lambda=0.001, \gamma_{0}=0.0007, K_{1}=0.07, k_{d}=0.25$,

$P e=0.7, \alpha_{0}=2.0 \mathrm{~km}, D^{*}=0.000015 \mathrm{~km}^{2} /$ year,

$n=0.32$ gravel, 0.55 clay, $m=0.04$ year $^{-1}$

The non-dimensional length of the aquifer was considered in the range $0<X \leq 0.1$. The concentration pattern is depicted for $1,1.5$ and 2 years, respectively. The source was assumed to be activated from 1 year in the domain of geological formation. The effect of source contaminant was predicted for the regular interval of six months. The effects of the various parameters are shown by the graph. The different velocity patterns are also revealed through Fig. 1 to Fig. 3.

The distribution pattern of the contaminant concentration was high in the aquitard compared to the aquifer for the same time interval and ultimately it went to its minimum harmless concentration with distance. The exponentially decreasing form of velocity with mean time in the direction of flow i.e., longitudinal direction, is described in Fig. 4. Fig. 5 shows for the sinusoidally varying form of the velocity pattern, where the distribution pattern of the concentration decreases with decreasing values of dispersivity $Q$. The dispersivity effect with respect to concentration levels is predicted up to a distance of $1.0 \mathrm{~km}$ in the domain. From Fig. 5, it is clear that the concentration

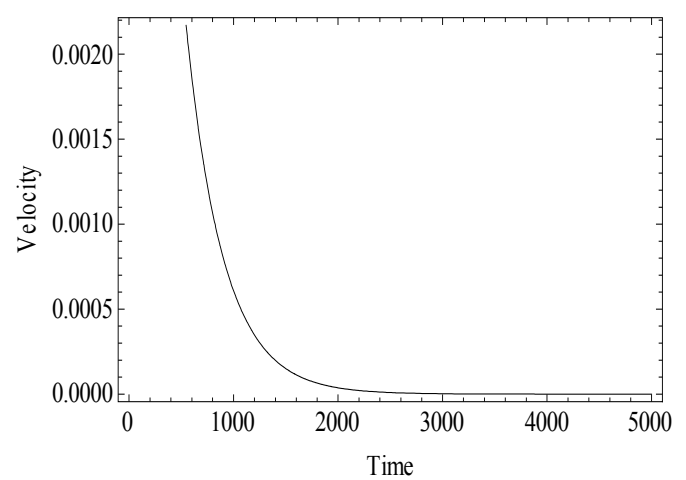

Fig. 1. Exponentially decreasing velocity pattern. 


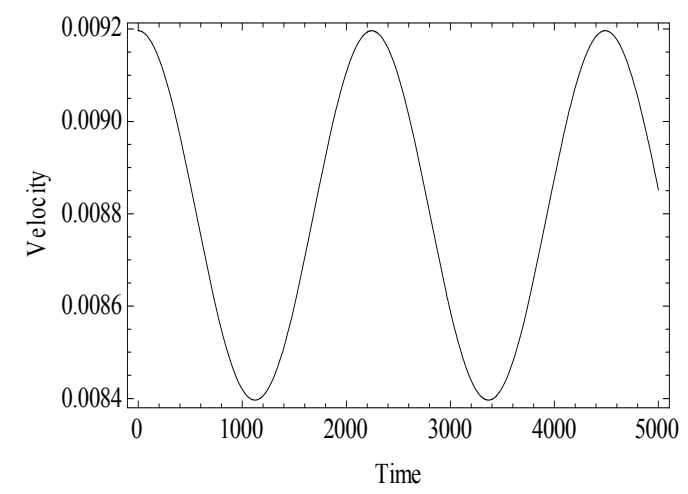

Fig. 2. Sinusoidally varying velocity pattern.

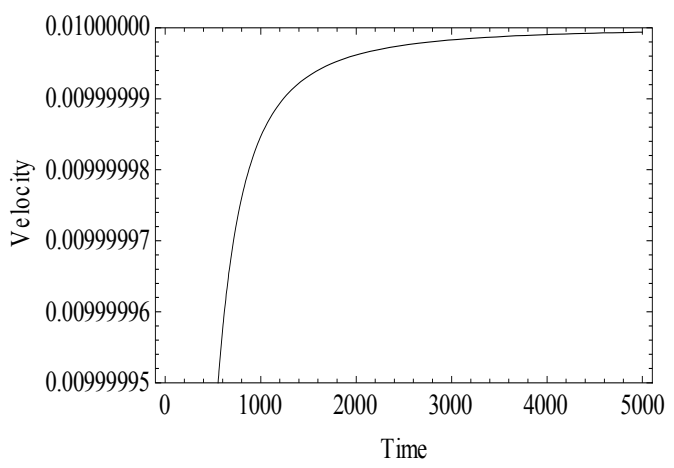

Fig. 3. Algebraic sigmoid velocity pattern.

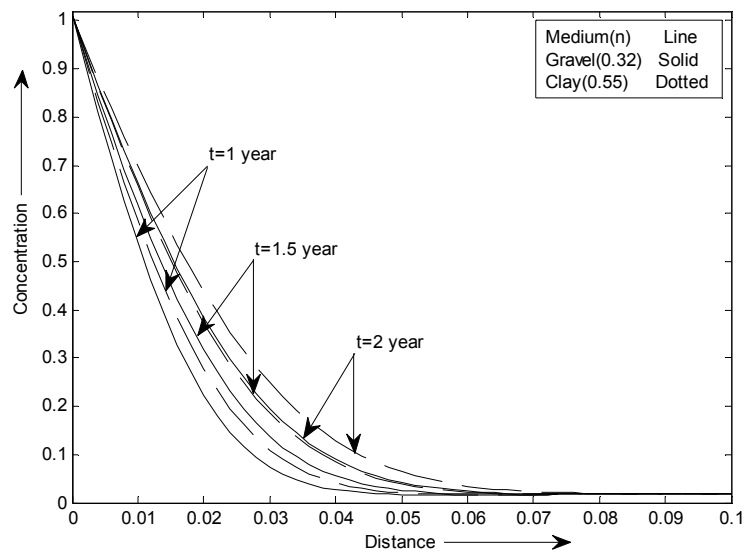

Fig. 4. The concentration distribution pattern for space dependent dispersion with exponentially decreasing velocity.

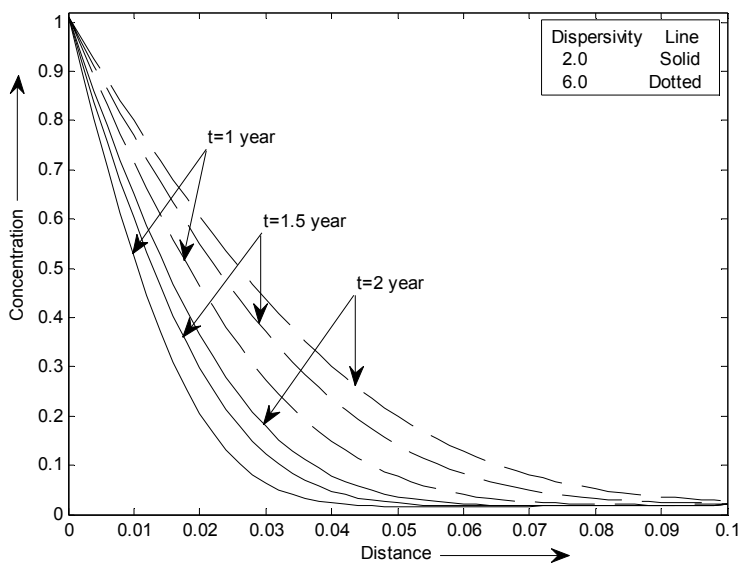

Fig. 5. The concentration distribution pattern for space dependent dispersion with sinusoidal form of velocity in aquifer (i.e., gravel) with respect to dispersivity. levels increase with increasing dispersivity of the solute. The algebraic sigmoid form of velocity depicts the contaminant concentration for different values of the mean travel time in the longitudinal direction and is revealed in Fig. 6. The contaminant concentration increases with decreasing values of the mean travel time. The mean travel time is particularly evaluated from the linear combination of dispersion and diffusion in the porous formation with longitudinal flow direction. So, if the value of the solute in the longitudinal direction slightly increases or decreases the mean travel time changes accordingly. The little variation for the source concentration is also observed only due to the time dependent source concentration taken into consideration at the origin of domain.

Different types of the velocity expression were considered for the pictorial representation of the obtained analytical solution given in equation (35) for the time dependent dispersion.

1) Exponential decreasing: $u=u_{0} \frac{\exp \left(-K_{1} m t\right)}{K_{1}}$ and $\tau=\frac{u_{0}^{2}}{K_{1}^{2} m D_{0}}\left[1-e^{-K_{1} m t}\right]$

2) Sinusoidal form: $u=u_{0} \frac{\left[4 K_{1} \pi+m \cos \left(K_{1} m t\right)\right]}{K_{1}}$ and $\tau=\frac{u_{0}^{2}}{D_{0} K_{1}^{2}}\left[4 K_{1}^{2} \pi t+\sin \left(K_{1} m t\right)\right]$

3) Algebraic sigmoid: $u=u_{0} \frac{1}{K_{1}}\left[\frac{m t}{\sqrt{(m t)^{2}+K_{1}^{2}}}\right]$ and $\tau=\frac{u_{0}^{2}}{m D_{0} K_{1}}\left[\sqrt{(m t)^{2}+K_{1}^{2}}-K_{1}\right]$

For graphical representation, the one-dimensional analytical solution of the homogeneous advection dispersion equation with the time-dependent dispersion given in equation (35) was computed for the set of input data:

$c_{i}=0.01, c_{0}=1.0, u_{0}=0.01 \mathrm{~km} /$ year,$D_{0}=0.1 \mathrm{~km}^{2} /$ year,

$\lambda=0.001, \gamma_{0}^{*}=0.0007, k_{d}=0.25, \mu_{0}^{*}=0.0001, K_{1}=0.07$,

$D^{*}=0.000015 \mathrm{~km}^{2} /$ year, $n=0.32$ gravel, 0.55 clay,

$m=0.04$ year $^{-1}$

The length of the domain for the time-dependent dispersion was taken $0 \leq X \leq 0.1$. The source of contaminant was predicted for the same time intervals: $1,1.5$ and 2 years, respectively, as discussed earlier.

From Fig. 7, it is clear that the concentration values near the source decrease with time in the aquitard as compared to the aquifer, but the trend is just reverse after some distance say 0.01 . This may happen due to the inclusion of mean travel time in the velocity expression. It is also depicted that the concentration values decrease with distance in both the geological formations. The level of contaminant concentration is observed slightly higher in the time-dependent dispersion as compared to space-dependent dispersion. This can be shown through Fig. 4 and Fig. 7.

The contaminant concentration values are different for different values of zero order production term in the sinusoidal form of velocity. The fluctuation of the source concentration in the domain is observed due to time-dependent source input concentration. The concentration level for the different time intervals decreased near the source and approximately up to some distance but after damping, it showed the reverse pattern 


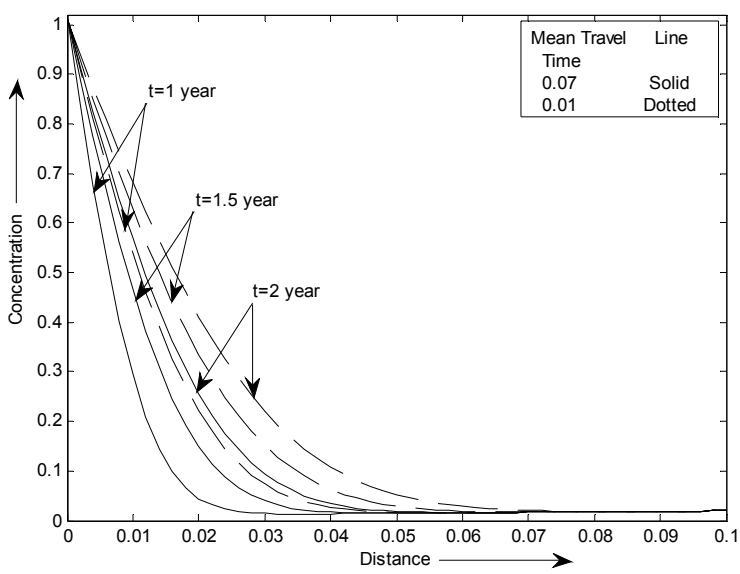

Fig. 6. The concentration distribution pattern for space dependent dispersion with algebraic sigmoid form of velocity in aquitard (i.e., clay) with respect to mean travel time.

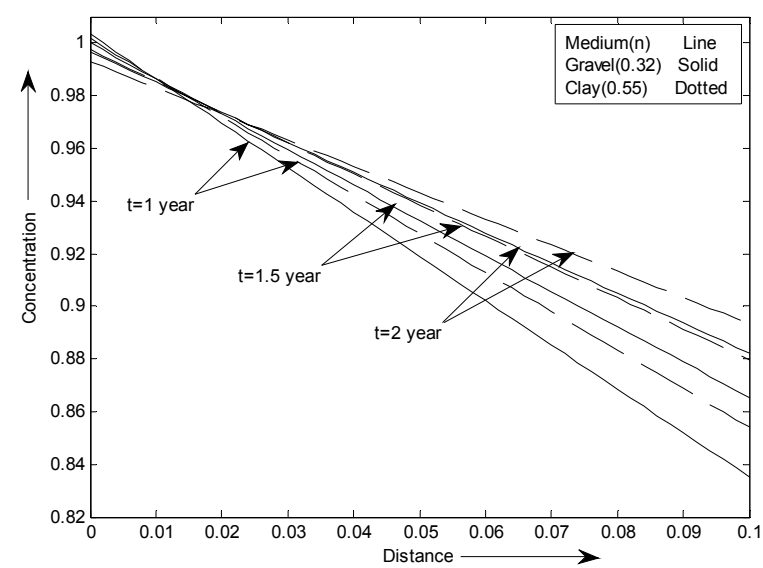

Fig. 7. The concentration distribution pattern for time dependent dispersion with exponentially decreasing form of velocity in aquifer (i.e., gravel) and aquitard (i.e., clay).

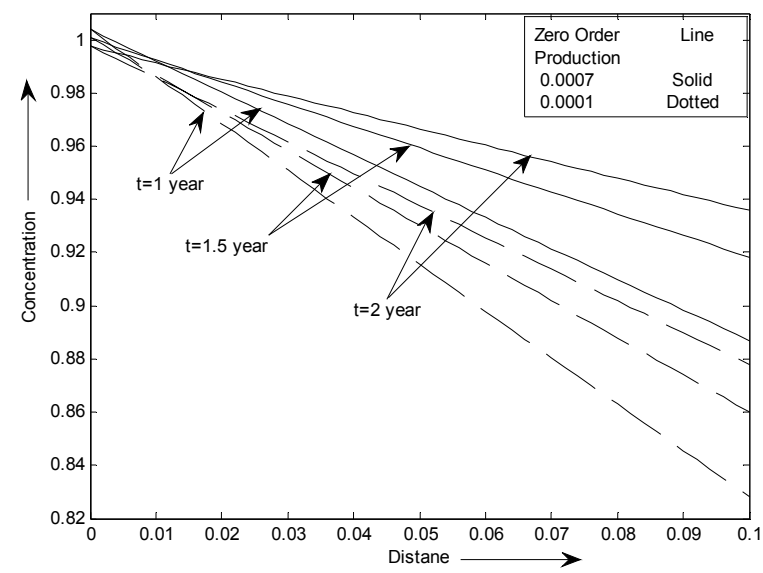

Fig. 8. The concentration distribution pattern for sinusoidal form of velocity in aquifer (i.e., gravel) with respect to the zero order production term.

i.e., the concentration level slightly increased with increasing time period beyond some distance. Then slowly the concentration level became stable with respect to distance. As we increased the value of zero order production term, the concentration values increased with their respective time, but ultimately the concentration level became stable after some distance.

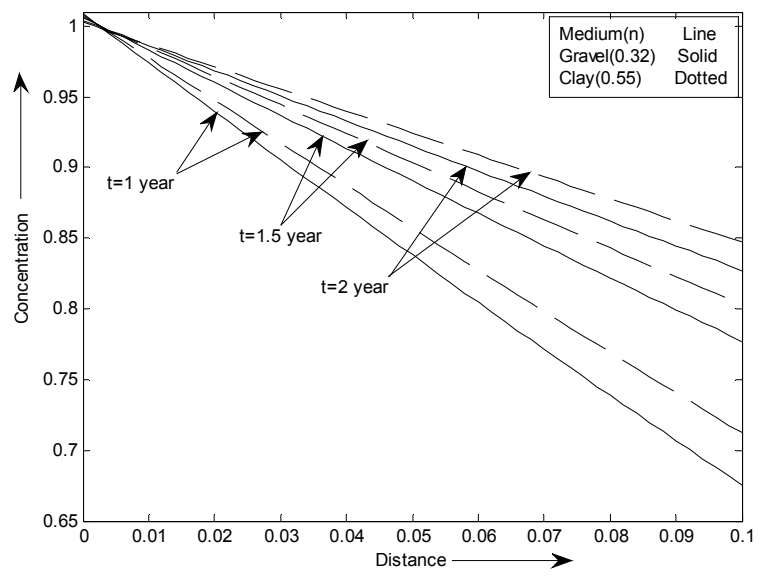

Fig. 9. The concentration distribution pattern for the algebraic sigmoid form of velocity in aquifer (i.e., Gravel) and aquitard (i.e., Clay).

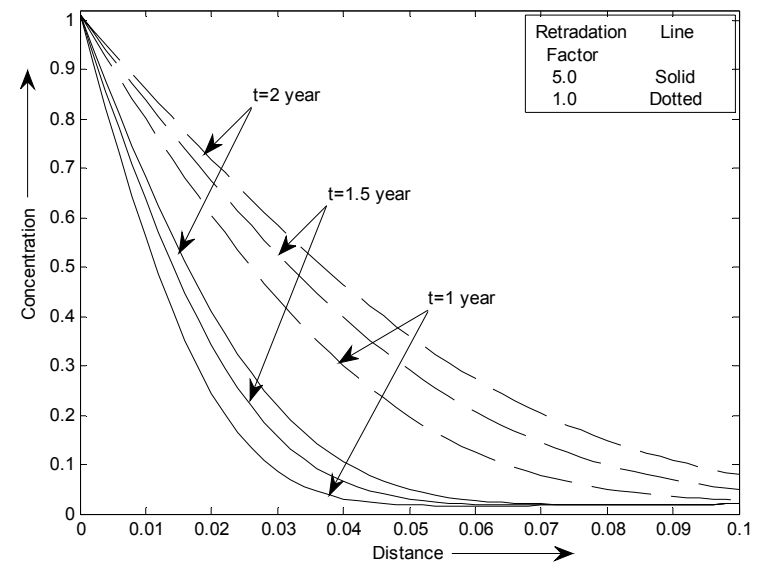

Fig. 10. The concentration distribution patterns for the constant dispersion with diffusion with respect to retardation factors.

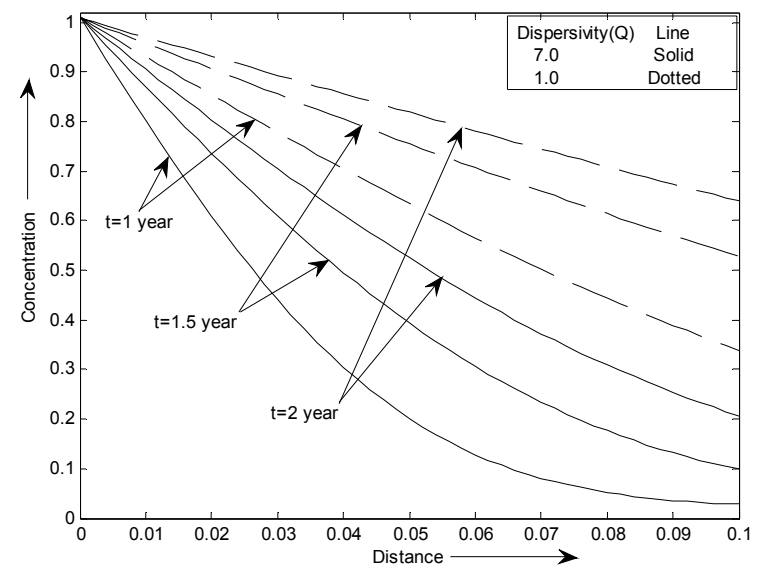

Fig. 11. The concentration distribution pattern for the linear dispersion with diffusion with respect to the dispersivity.

So, the fluctuation nature in the tropical region is described for the sinusoidal form of the velocity pattern in Fig.8.

In Fig.9, we have discussed the algebraic sigmoid form of the velocity pattern. We observed that the solute concentration increased in the aquitard where the transfer rate of the liquid was low as compared to the aquifer. So, the level of contaminant concentration pattern was minimum in the aquifer as compared to the aquitard. 


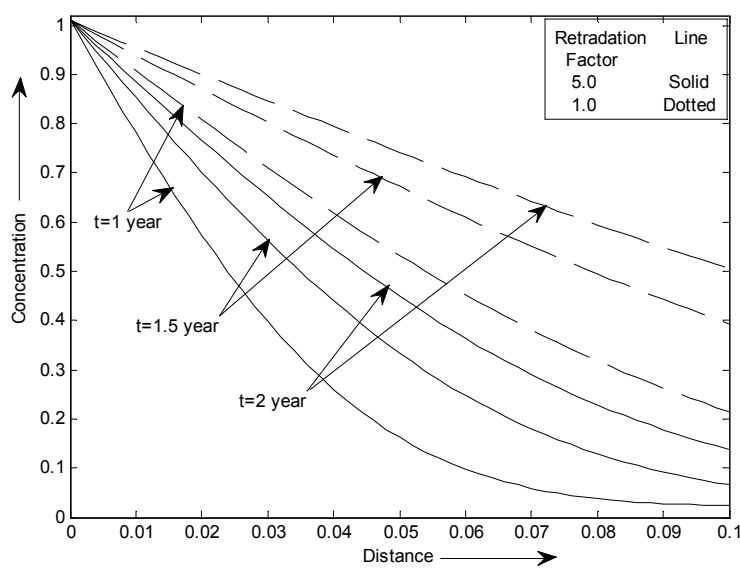

Fig. 12. The concentration distribution pattern for the exponential dispersion with diffusion with respect to the retardation factors.

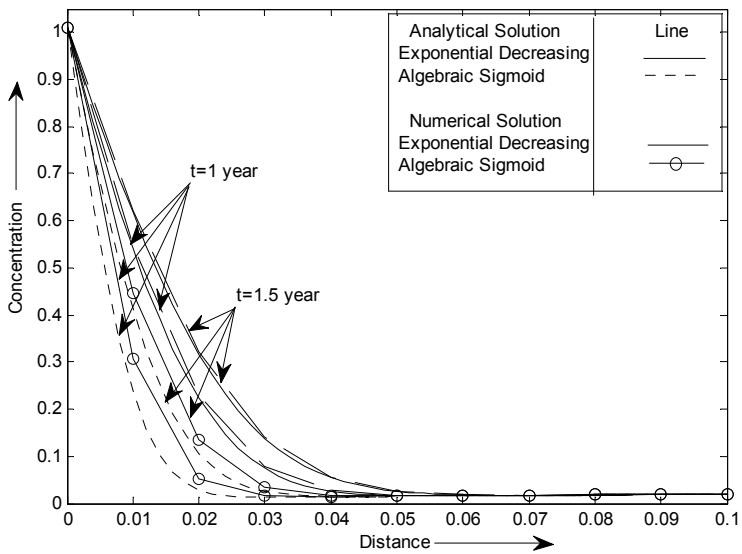

Fig. 13. Comparision between the concentration distribution pattern of the analytical numerical solution for the gravel medium.

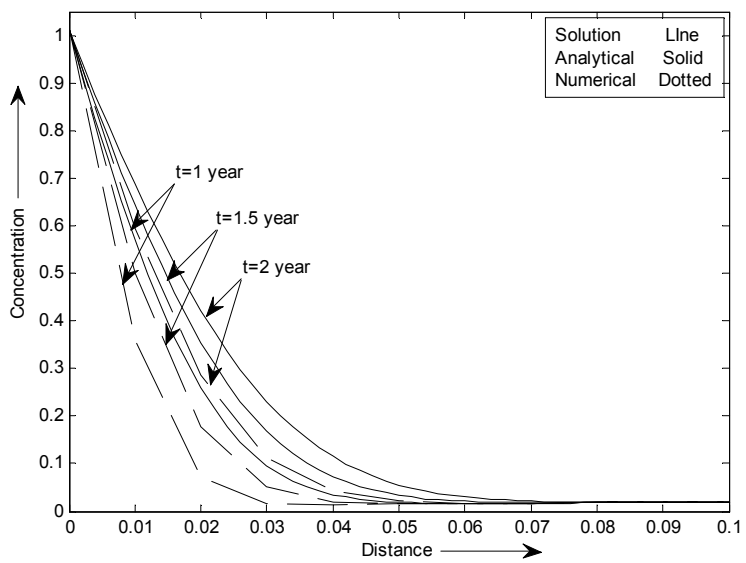

Fig. 14. Comparision between the concentration distribution pattern of the analytical and numerical solution for the clay medium along sinusoidal velocity pattern.

The time-dependent dispersion for the concentration distribution pattern was described only for the asymptotic type of dispersion (Basha and El-Habel, 1993). In view of this, we discussed the three different types of generalized cases for the time dependent function which are as follows:

1) Constant dispersion: $f(m t)=K_{1}$ and $\tau=\frac{u_{0}^{2} K_{1} t}{D_{0}}$

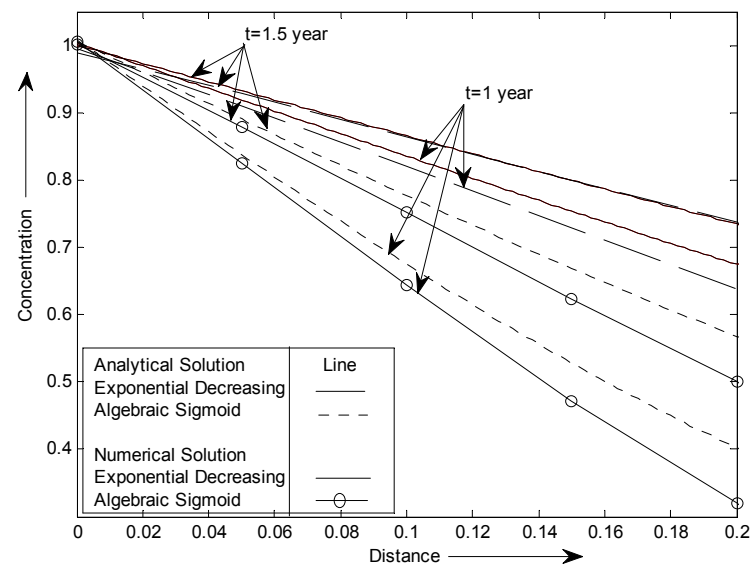

Fig. 15. Comparision between the concentration distribution pattern of the analytical and numerical solution for the gravel medium.

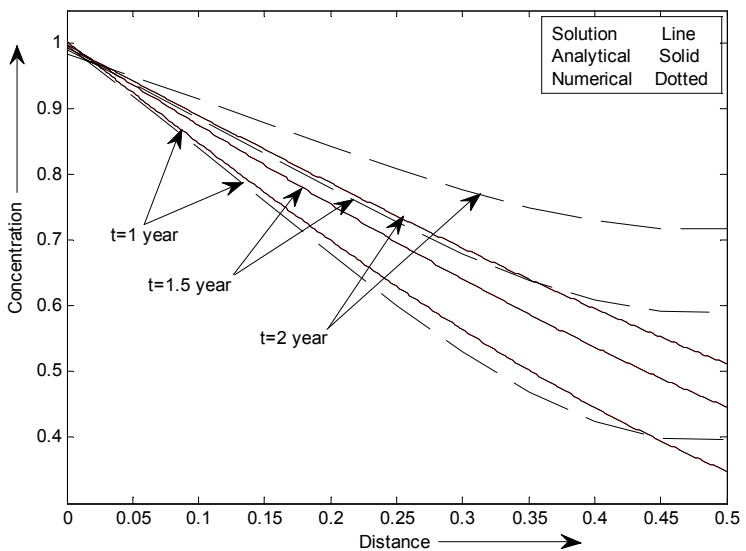

Fig. 16. Comparision between the concentration distribution pattern of the analytical and numerical solution for the clay medium along sinusoidal velocity pattern.

2) Linear dispersion: $f(m t)=t$ and $\tau=\frac{u_{0}^{2} t^{2}}{2 K_{1} D_{0}}$

3) Exponential: $\quad f(m t)=K_{1}\left[1-\exp \left(-\frac{t}{K_{1}}\right)\right]$ and $\tau=\frac{u_{0}^{2}}{D_{0}}\left[K_{1} t+\exp \left(-\frac{t}{K_{1}}\right)-1\right]$

The same input values are considered for the computation of the solution given in Eq. (35) with parameters like dispersivity $Q=1$, retardation factor $R=1$ and the diffusion coefficient $D^{*}=0$ which were taken by Basha and El-Habel (1993). Fig. 10 depicts the contaminant concentration for the constant type of dispersion with the same value that was already used for the time-dependent dispersion. In Fig. 10, we also explore the contaminant concentration for the constant type of dispersion for different values of the retardation factor. From this, we observed that the values of contaminant concentration decreased at each of the positions compared with the concentration values with respect to the retardation factor used by Basha and El-Habel (1993). In both cases, the concentration pattern attained its minimum concentration level after traveling some distance. The contaminant concentration pattern was also predicted for the linear and exponential types of dispersion in Fig. 11 and Fig. 12 with the mean travel time $K_{1}=D^{*}+Q$ and 
$K_{1}=0.632 Q+D^{*}$ (Basha and El-Habel, 1993), respectively. The contaminant concentration was predicted for the same set of data except $R=1, D^{*}=0, Q=1.7$, as shown in Fig. 11 . From this, we observed that the value of contaminant concentration decreased with increasing values of dispersivity with their respective time and attained its minimum concentration level with respect to their respective distances. Due to the exponentially decreasing term present in the exponential dispersion, the value of the dispersivity slightly changed from 1 to 1.5 for their respective mean travel times. From Fig.12, we observe that the contaminant concentration values for the exponential type of dispersion also decreased compared to the value of retardation factor taken by Basha and El-Habel (1993) at each of the positions.

In Figs. (13) and (14) the predicted concentration distribution pattern for the space-dependent diffusion is presented, whereas Figs. (15) and (16) show the predicted concentration distribution pattern for the time-dependent diffusion. The numerical result is predicted for the same set of input values which is taken for the analytical result. Comparison of the analytical result to the numerical one with different velocity patterns, and the concentration distribution pattern for the gravel medium with average porosity $(0.32)$ for different time duration, are shown in Fig. 13. The concentration values by the analytical solution for the exponential decreasing form of the velocity pattern yielded decreasing values at each of the positions as compared to the numerical solution. The concentration values increased at each of the positions with increasing time duration for both solutions i.e., analytical and numerical. Similar types of contaminant concentration were depicted for the algebraic sigmoid form of the velocity pattern which showed the fluctuation pattern of groundwater after rainy seasons. From both the solutions, we observed that concentration values were higher in the case of the exponential decreasing velocity pattern as compared to the algebraic sigmoid form of the velocity for both time durations. The contaminant concentration decreased with distance and increased with time. From Fig. 14 the minimum concentration values were observed at each of the positions in the case of numerical solution as compared to the analytical solution for the sinusoidal form of the velocity pattern for the clay medium with the average porosity of 0.32 . The concentration values increased with time and decreased with distance for both the analytical and numerical. Fig.15 follows the reverse nature of the concentration distribution for different velocities in the case of analytical and numerical solutions of time-dependent diffusion as in Fig.13. Analytical solutions yielded high concentration values as compared to the numerical solution for both exponentially decreasing and algebraic sigmoid forms of the velocity pattern. In the case of $t=1.5$ years, the numerical solution for exponentially decreasing velocity forms initially produces less concentration values than did the analytical solution. However, after some distance the concentration values were observed to be approximately the same for both the solutions. We also observed from Fig. 15 that the concentration values were higher for the exponentially decreasing form of velocity than for the algebraic sigmoid form of the velocity pattern for both the solutions. The concentration distribution for the sinusoidal form of the velocity pattern is depicted in Fig. 16. We observed that initially the source of concentration was the same for the entire duration of time, but in the first year the numerical solution gave lower concentration values than did the analytical one and after some distance it gave the reverse pattern. For the next 1.5 and 2 years the concentration distribution pattern followed the reverse pattern. In the respective years the numerical solution gave higher concentration values than did the analytical solution. Also the numerical solutions followed the uniform nature of the decreasing concentration pattern after covering some distance. Finally from Fig. 16 , it is observed that at the end of the domain the concentration values were higher in the case of numerical solution than for the analytical solution.

RMSE of clay and gravel media for the fixed time duration i.e., 2 years are tabulated for the space-dependent dispersion with diffusion, as shown in Tables 1(a) and 1(b), respectively. However, RMSE of the same is tabulated for the timedependent dispersion with diffusion, as shown in Tables 2(a) and 2(b), respectively. Tables 1 (a) and 1(b) are tabulated for the fixed space and time increments i.e., $\Delta X^{\prime}=0.01, \Delta T=0.0001$, however, Tables 2(a) and 2(b) are tabulated for the fixed space and time increments, i.e., $\Delta X^{\prime}=0.05, \Delta \tau=0.0001$. It is observed from Table1 (a) that the RMSE value is minimum for the exponentially decreasing and sinusoidal forms unlike the algebraic sigmoid form of the velocity patterns of the clay medium. Similar observations were made for the gravel medium with a fixed time duration of 2 years. From Table 1 (a) and 1 (b), it is clear that the RMSE values were lower in the aquifer (i.e., gravel) medium than in the aquitard (i.e., clay) medium for different respective velocity patterns.

From Table 2 (a) and (b) the RMSE values were minimum for the algebraic sigmoid but maximum for the exponentially

Table 1(a). RMSE of the clay medium for average porosity $(0.55)$ with 2 years duration for scale dependent dispersion with diffusion.

\begin{tabular}{|c|c|c|c|c|c|c|}
\hline \multicolumn{3}{|c|}{ Exponential decreasing } & \multicolumn{2}{|c|}{ Sinusoidal form } & \multicolumn{2}{|c|}{ Algebraic sigmoid } \\
\hline Distance & Analytical result & Numerical result & Analytical result & Numerical result & Analytical result & Numerical result \\
\hline 0.02 & 0.4391 & 0.4407 & 0.4209 & 0.4295 & 0.2559 & 0.2852 \\
\hline 0.04 & 0.1289 & 0.1267 & 0.1150 & 0.1181 & 0.0343 & 0.0418 \\
\hline 0.06 & 0.0344 & 0.0326 & 0.0302 & 0.0301 & 0.0164 & 0.0169 \\
\hline 0.08 & 0.0196 & 0.0186 & 0.0190 & 0.0183 & 0.0179 & 0.0177 \\
\hline 0.10 & 0.0199 & 0.0178 & 0.0199 & 0.0178 & 0.0199 & 0.0182 \\
\hline RMSE & \multicolumn{2}{|c|}{0.00179} & \multicolumn{2}{|c|}{0.00420} & \multicolumn{2}{|c|}{0.01354} \\
\hline
\end{tabular}

Table 1(b). RMSE of the gravel medium for average porosity $(0.32)$ with 2 years duration for scale dependent dispersion with diffusion.

\begin{tabular}{|c|c|c|c|c|c|c|}
\hline \multicolumn{3}{|c|}{ Exponential decreasing } & \multicolumn{2}{c|}{ Sinusoidal form } & \multicolumn{2}{c|}{ Algebraic sigmoid } \\
\hline Distance & Analytical result & Numerical result & Analytical result & Numerical result & Analytical result & Numerical result \\
\hline 0.02 & 0.3844 & 0.3857 & 0.3655 & 0.3741 & 0.2018 \\
\hline 0.04 & 0.0903 & 0.0895 & 0.0791 & 0.0827 & 0.2307 \\
\hline 0.06 & 0.0239 & 0.0236 & 0.0217 & 0.0223 & 0.0229 & 0.0160 \\
\hline 0.08 & 0.0183 & 0.0178 & 0.0181 & 0.0177 & 0.0179 \\
\hline 0.10 & 0.0199 & 0.0179 & 0.0199 & 0.0179 & 0.0199 \\
\hline RMSE & \multicolumn{2}{|c|}{0.00115} & \multicolumn{3}{|c|}{0.00427} & 0.0177 \\
\hline
\end{tabular}


Mathematical modeling of groundwater contamination with varying velocity field

Table 2(a). RMSE of the clay medium for average porosity (0.55) with 2 years duration for time dependent dispersion with diffusion.

\begin{tabular}{|c|c|c|c|c|c|c|}
\hline \multicolumn{3}{|c|}{ Exponential decreasing } & \multicolumn{2}{c|}{ Sinusoidal form } & \multicolumn{2}{c|}{ Algebraic sigmoid } \\
\hline Distance & Analytical result & Numerical result & Analytical result & Numerical result & Analytical result & Numerical result \\
\hline 0.1 & 0.8934 & 0.9213 & 0.8899 & 0.9145 & 0.8469 \\
\hline 0.2 & 0.7951 & 0.8587 & 0.7870 & 0.8427 & 0.8384 \\
\hline 0.3 & 0.7004 & 0.8012 & 0.6883 & 0.7766 & 0.676 \\
\hline 0.4 & 0.6113 & 0.7600 & 0.5960 & 0.7294 & 0.5612 \\
\hline 0.5 & 0.5296 & 0.7487 & 0.5120 & 0.7165 & 0.4429 \\
\hline RMSE & \multicolumn{2}{|c|}{0.13046} & \multicolumn{2}{c|}{0.11926} & 0.4179 & 0.385 \\
\hline
\end{tabular}

Table 2(b). RMSE of the gravel medium for average porosity $(0.32)$ with 2 years duration for time dependent dispersion with diffusion.

\begin{tabular}{|c|c|c|c|c|c|c|}
\hline \multicolumn{3}{|c|}{ Exponential decreasing } & \multicolumn{2}{c|}{ Sinusoidal form } & \multicolumn{2}{c|}{ Algebraic sigmoid } \\
\hline Distance & Analytical result & Numerical result & Analytical result & Numerical result & Analytical result & Numerical result \\
\hline 0.1 & 0.8822 & 0.8946 & 0.8778 & 0.8867 & 0.8270 \\
\hline 0.2 & 0.7699 & 0.8041 & 0.7606 & 0.7858 & 0.8086 \\
\hline 0.3 & 0.6631 & 0.7209 & 0.6495 & 0.6930 & 0.6592 \\
\hline 0.4 & 0.5645 & 0.6613 & 0.5477 & 0.6266 & 0.5098 \\
\hline 0.5 & 0.4761 & 0.6454 & 0.4574 & 0.6089 & 0.3853 \\
\hline RMSE & \multicolumn{2}{|c|}{0.09240} & \multicolumn{3}{c|}{0.07972} & 0.2882 \\
\hline
\end{tabular}

decreasing form of the velocity pattern. The aquifer (i.e., gravel) had minimum values of RMSE other than the aquitard (i.e. clay) medium for different respective velocity patterns except the algebraic sigmoid form. The RMSE was minimum for clay as compared to gravel for the algebraic sigmoid form of the velocity pattern. So, the RMSE values were close for the gravel medium for both space- and time-dependent diffusion (except algebraic sigmoid). For space-dependent diffusion RMSE was very close for the exponential decreasing velocity, whereas in the case of time-dependent diffusion result the closest values were found for the algebraic sigmoid form of the velocity pattern.

\section{SUMMARY AND CONCLUSION}

Based on the present study two analytical models were developed for one-dimensional solute transport in the semi-infinite domain with the distance-dependent and timedependent dispersivities. The level of the contaminant concentration is predicted for the aquifer and aquitard. The result is also compared with input parameters considered by Basha and El-Habel (1993). We can conclude as follows:

1. Analytical solutions are obtained using LITT for space-dependent as well as time-dependent dispersion with diffusion which are useful to benchmark numerical codes and solutions. These solutions may be applicable as a preliminary predictive tool in groundwater resource and management.

2. Analytical results are compared with numerical ones and the agreement between them is found to be very good. The accuracy of results is verified by RMSE values.

3. The concentration distribution is obtained for different geological formations, such as aquifer and aquitard, with varying velocity field and it is found that the concentration values are less in aquifer than in aquitard.

Acknowledgement. The authors are thankful to Prof. Vijay P Singh, Distinguished Professor and Caroline \& William N. Lehrer Distinguished Chair in Water Engineering, Department of Biological and Agricultural Engineering \& Zachry Dept. Civil Engineering, Texas A and M University, Texas, USA for language editing. The authors are thankful to the editor and the reviewers whose constructive comments helped improve the quality of the paper. The authors are also thankful for providing financial support to Ph.D. candidate under the ISMJRF scheme. This work is partially supported by the CSIR Project no. 25(0251)/16/EMR-II.

\section{REFERENCES}

Aral, M.M., Liao, B., 1996. Analytical solutions for twodimensional transport equation with time-dependent dispersion coefficients. J. Hydrol. Eng., 1, 20-32.

Barry, D.A., Sposito, G., 1989. Analytical solution of a convection-dispersion model with time-dependent transport coefficients. Water Resour. Res., 25, 2407-2416.

Basha, H.A., El-Habel, F.S., 1993. Analytical solution of the one-dimensional time dependent transport equation. Water Resour. Res., 29, 3209-3214.

Batu, V., 2006. Applied Flow and Solute Transport Modeling in Aquifers: Fundamental Principles and Analytical and Numerical Methods. CRC, Boca Raton, FL.

Bear, J., 1972. Dynamics of Fluids in Porous Media. Elsevier, New York, USA.

Carnahan, B., Luther, H.A., Wilkes, J.O., 1969. Applied Numerical Methods. Wiley, New York, pp. 429-530.

Chaudhari, N.M., 1971. An improved numerical technique for solving multidimensional miscible displacement equation. Soc. Petrol. Engng J., 11, 277-284.

Chen, J.S., Lai, K.H., Liu, C.W., Ni, C.F., 2012. A novel method for analytically solving multi-species advectivedispersive transport equations sequentially coupled with first-order decay reactions. J. Hydrol., 420-421, 191-204.

Crank, J., 1975. The Mathematics of Diffusion. 2nd ed. Oxford Univ. Press, London.

De Smedt, F., Wierenga, P.J., 1977. Simulation of water and solute transport in unsaturated soils. In: Proc. 3rd Int. Symp. on Hydrology, Fort Collins. CO.

Dudley, L.M., McLean, J.E., Furat. T.H., Jurinak, J.J., 1991. Sorption of cadmium copper from an acid mine waste extract by two calcareous soils: column studies. Soil Sci., 151, 121-135.

Ebach, E.H., White, R., 1958. Mixing of fluids flowing through beds of packed solids. J. American Institute of Chemical Eng., 4, 161-164.

Freeze, R.A., Cherry, J.A., 1979. Groundwater. Prentice-Hall, Englewood Cliffs, New Jersey.

Fried, J.J., Combarnous, M.A., 1971. Dispersion in porous media. Adv. Hydrosci., 7, 169-281.

Gelhar, L.W., Welty, W., Rehfeldt, K.R., 1992. A critical review of data on field-scale dispersion in aquifers. Water Resour. Res., 28, 1955-1974.

Ghosh, N.C., Sharma, K.D., 2006. Groundwater Modelling and Management. Capital Publishing Company, New-Delhi. 
Gillham, R.W., Cherry, J.A., 1982. Contaminant migration in saturated unconsolidated geologic deposits. Geological Society of America, Special Paper, 189, 31-62.

Guerrero, J.S.P., Skaggs, T.H., 2010. Analytical solution for one-dimensional advection-dispersion transport equation with distance-dependent coefficients. J. Hydrol., 390, 57-65.

Guerrero, J.S.P., Pontedeiro, E.M., van Genuchten, M.T., Skaggs, T.H., 2013. Analytical solutions of the onedimensional advection-dispersion solute transport equation subject to time-dependent boundary conditions. Chemical Eng. J., 221, 487-491.

Huang, K., van Genuchten, M.T., Zhang, R., 1996. Exact solutions for one-dimensional transport with asymptotic scaledependent dispersion. Appl. Math. Modelling, 20, 298-308.

Huang, G., Huang, Q., Zhan, H., 2006. Evidence of onedimensional scale-dependent fractional advection dispersion. J. Contam. Hydrol., 85, 53-71.

Jain, S.K., Agarwal, P.K., Singh, V.P., 2007. Hydrology and Water Resources of India. Springer, The Netherlands, p. 87.

Lantz, R.B., 1971. Quantitative evaluation of numerical diffusion truncation error. Society Petrole. Engg. J., 11, 315-320.

Mickley, H.S., Sherwood, T.K., Reed, C.E., 1957. Applied Mathematics in Chemical Engineering. McGraw-Hill, New York

Mitchell, J.K., 1976. Fundamentals of Soil Behavior. Wiley, New York.

Moldrup, P., Paulsen, T.G., Rolston, D.E., Yamaguchi, T., Hansen, J.A., 1994. Integrated flux model for unsteady transport of trace organic chemicals in soils. Soil Sci., 157, $137-152$.

Notodarmojo, S., Ho, G.E., Scott, W.D., Davis, G.B., 1991. Modelling phosphorus transport in soils and groundwater with two-consecutive reactions. Water Res., 25, 10, 1205-1216.

Ogata, A., Banks, R.B., 1961. A solution of differential equation of longitudinal dispersion in porous media. Geological Survey Professional Paper, 411-A.

Roberts, D.L., Selim, M.S., 1984. Comparative study of six explicit and two implicit finite difference schemes for solving one-dimensional parabolic partial differential equations. Int. J. Numer. Methods Eng., 20, 817-844.

Scheidegger, A.E., 1957. The Physics of Flow through Porous Media. University of Toronto Press, Toronto.

Scheidegger, A.E., 1961. General theory of dispersion in porous media. J. Geophys. Res., 66, 10, 3273-3278.

Sharma, H.D., Reddy, K.R., 2004. Geo-Environmental Engineering. Wiley, New York.

Singh, P., Singh, V.P., 2001. Snow and Glacier Hydrology. Kluwer Academic Publishers, Amsterdam, The Netherlands, p. 78.

Singh, M.K., Singh, V.P., Singh, P., Shukla, D., 2009. Analytical solution for conservative solute transport in onedimensional homogeneous porous formation with timedependent velocity. J. Engg. Mech., 135, 9, 1015-1021.

Singh, M.K., Ahamad, S., Singh, V.P., 2012. Analytical solution for one-dimensional solute dispersion with timedependent source concentration along uniform groundwater flow in a homogeneous porous formation. J. Eng. Mech., $138, \quad 8, \quad 1045-1056$, DOI: 10.1061/(ASCE)EM.19437889.0000384 .

Smith, G.D., 1978. Numerical Solution of Partial Differential Equations: Finite Difference Methods. 2nd ed. Oxford University Press, Oxford.

Towler, B.F., Yang, R.Y.K., 1979. On comparing the accuracy of some finite-difference methods for parabolic partial differential equations. Int. J. Numer. Methods Eng., 14, 10211035 . van Genuchten, M.T., Gray, W.G., 1978. Analysis of some dispersion corrected numerical schemes for solution of the transport equation. Int. J. Num. Methods Eng., 12, 387-404.

van Genuchten, M.T., Leij, F.J., Skaggs, T.H., Toride, N., Bradford, S.A., Pontedeiro, E.M., 2013. Exact analytical solutions for contaminant transport in rivers. 1. The equilibrium advection-dispersion equation. J. Hydrol. Hydromech., $61,2,146-160$.

You, K., Zhan, H., 2013. New solutions for solute transport in a finite column with distance-dependent dispersivities and time-dependent solute sources. J. Hydrol., 487, 87-97.

Zamani, K., Bombardelli, F.A., 2014. Analytical solutions of nonlinear and variable-parameter transport equations for verification of numerical solvers. Environ. Fluid Mech., 14, 711-742. DOI: 10.1007/s10652-013-9325-0.

\section{NOMENCLATURE}

The following symbols are used in this paper:

$D \quad$ Longitudinal dispersion coefficient; $\left[\mathrm{L}^{2} \mathrm{~T}^{-1}\right]$

$D^{*} \quad$ Molecular diffusion coefficient; $\left[\mathrm{L}^{2} \mathrm{~T}^{-1}\right]$

$c \quad$ The volume averaged dispersing solute concentration in the liquid phase; $\left[\mathrm{ML}^{-3}\right]$

$F \quad$ The volume averaged dispersing solute concentration in the solid phase; $\left[\mathrm{ML}^{-3}\right]$

$c_{i} \quad$ Initial concentration; $\left[\mathrm{ML}^{-3}\right]$

$c_{0} \quad$ Source concentration; $\left[\mathrm{ML}^{-3}\right]$

$\lambda \quad$ The decay rate constant; $\left[\mathrm{T}^{-1}\right]$

$u \quad$ Unsteady uniform pore seepage velocity; $\left[\mathrm{LT}^{-1}\right]$

$K_{d} \quad$ Distribution coefficient

$D_{0} \quad$ Initial dispersion coefficient; $\left[\mathrm{L}^{2} \mathrm{~T}^{-1}\right]$

$u_{0} \quad$ Initial seepage velocity; $\left[\mathrm{LT}^{-1}\right]$

$n \quad$ Porosity of the different geological formation

$x \quad$ The longitudinal direction of flow; [L]

$m \quad$ The flow resistance coefficient; $\left[\mathrm{T}^{-1}\right]$

$t \quad$ Time variable; [T]

$T^{*} \quad$ New time variable; $[\mathrm{T}]$

$\tau^{*} \quad$ New time variable; $[\mathrm{T}]$

$\mu \quad$ First order decay rate coefficients; $\left[\mathrm{T}^{-1}\right]$

$\gamma \quad$ Zero order production rate coefficient; $\left[\mathrm{ML}^{-3} \mathrm{~T}^{-1}\right]$

$R \quad$ Retardation factor

$P e \quad$ Peclet number

$Q \quad$ Maximum dispersivity; [L]

$K_{1} \quad$ The mean travel time; [T]

$f(m t)$ The generalised case of the time-dependent function

$c_{1}, c_{2} \quad$ Arbitrary constants

$\mu_{0} \quad$ Initial first order decay rate coefficients; $\left[\mathrm{T}^{-1}\right]$

$\mu_{0}^{*} \quad$ Initial first order decay rate coefficients; $\left[\mathrm{T}^{-1}\right]$

$\gamma_{0} \quad$ Initial zero order production rate; $\left[\mathrm{ML}^{-3} \mathrm{~T}^{-1}\right]$

$\gamma_{0}^{*} \quad$ Initial zero order production rate; $\left[\mathrm{ML}^{-3} \mathrm{~T}^{-1}\right]$

$q \quad$ Growing rate of dispersivity along the space

$\alpha_{0} \quad$ Asymptotic dispersivity; [L]

Received 7 April 2016 Accepted 8 September 2016 